\title{
Guías alimentarias y su cumplimiento en estudiantes universitarias, Valparaíso, 2013, Chile
}

\author{
Dietary guidelines and compliance \\ in college students, \\ Valparaíso, 2013, Chile
}

\begin{abstract}
Increased cardiovascular risk factors in young women merit knowing their nutritional profile. The objective of this study was to determine the food consumption of 50 volunteer college students enrolled in physical education (PE), early childhood education (ECA) and nutrition and dietetics (NAD) at the University of Playa Ancha, Valparaiso, Chile. A food frequency questionnaire was used to measure critical nutrient intake (sodium, sugars, saturated fatty acids, trans-fatty acids, cholesterol). Cardiovascular risk factors like alcohol intake were also measured as well as protective nutrients intake (mono and polyunsaturated fatty acids, water, dietary fiber). Consumption frequency was ranked high, moderate and low. Critical and protective nutrients were compared with the Chilean Dietary Guidelines 2013. Results: PE students' diet was high in sodium and dietary fiber; PE and EPA diet was high in added sugars and low in water; the diet of all groups was low in mono and polyunsaturated fatty acids, moderate in saturated fatty acids, trans-fatty acids and cholesterol, and low in alcohol. We conclude that the diets do not meet the recommendations of the Dietary Guidelines.

Key words: food habits, nutrients, university students, cardiovascular risk factors.
\end{abstract}

Mirta Crovetto M. (1) Bárbara Figueroa R. (2) Lisette González M. (2) Alejandra Jeria J. (2) Nicole Ramírez V. (2)

(1) Departamento de Nutrición, Centro de Estudios Avanzados,
Facultad de Ciencias de la Salud, Universidad de Playa Ancha, Valparaíso, Chile.
(2) Departamento de Nutrición, Facultad de Ciencias de la Salud,
Universidad de Playa Ancha, Valparaíso, Chile
Dirigir la correspondencia a:
Profesora
Mirta Crovetto M.
Departamento de Nutrición
Centro de Estudios Avanzados
Facultad de Ciencias de la Salud,
Universidad de Playa Ancha
Avenida Playa Ancha 890
Valparaíso, Chile
Fono 32-2500228
E-mail: mirtacrovetto@gmail.com; mcrovetto@upla.cl

Este trabajo fue recibido el 9 de Septiembre de 2014 y aceptado para ser publicado el 23 de Febrero de 2015.

\section{INTRODUCCIÓN}

La transición demográfica y epidemiológica de Chile se ha manifestado en un aumento de las Enfermedades Crónicas No Trasmisibles (ECNT) y de los Factores de Riesgo Cardiovascular (FRCV), los que se encuentran asociados a los estilos de vida, como hábitos alimentarios inadecuados los que se caracterizan por el consumo excesivo de nutrientes perjudiciales para la salud Cardiovascular (CV) denominados nutrientes críticos, como los Ácidos Grasos Saturados (AGS), el colesterol, los Ácidos Grasos Trans (AG Trans), el sodio y los azúcares, (1-3) principales causantes de dislipidemia (DLP), hipertensión arterial (HTA) y diabetes mellitus (DM), además por el bajo consumo de nutrientes protectores que benefician la salud CV los que contrarrestan los factores de riesgo de ECNT's, como son los ácidos grasos mono y polinsaturados (AGMI-AGPI), el agua y la fibra dietética $(1,4)$.

La Encuesta Nacional de Salud 2010 (ENSA, 2010), mostró que los mayores de 17 años presentan un predominio de las ECNT, las que se manifiestan a más temprana edad $(5,6)$. Estudios realizados en estudiantes universitarios en el país
$(7,8)$ indican que durante el período universitario pueden adquirirse hábitos poco saludables que influyen en la aparición de sobrepeso (SP) y obesidad (OB) y a consecuencia ECNT's. Al contrastar los resultados de la Encuesta Nacional de Salud 2003 (ENS, 2003) con la ENSA 2010, se observa un aumento en el SP de $21,9 \%$ a $25,1 \%$, y de OB de $37,8 \%$ a $39,3 \%$ y de $1,2 \%$ en $O B$ mórbida a $2,3 \%$ respectivamente mayor a un menor nivel educacional y en mujeres que en hombres. Cabe señalar que $75 \%$ de las cirugías bariátricas en Chile se realizan en mujeres $(6,8,9)$.

La Organización Mundial de la Salud (OMS) señala que 1,9 millones de muertes anuales son ocasionadas por la inactividad física, mientras que 2,7 millones son atribuibles a una ingesta insuficiente de frutas y verduras sumado al efecto de la obesidad con variados tipos de cánceres (CA) y ECNT. En este aspecto, se considera que hábitos alimentarios adecuados pueden reducir la incidencia delas ECNT con una dieta abundante en leguminosas, cereales integrales, frutas y hortalizas, pescados, mariscos y agua, y reducida en carnes rojas, alimentos procesados ricos en grasas saturadas y alcohol $(10,11)$. 
Los cambios en los hábitos alimentarios se asocian entre otros factores a la selección de alimentos poco saludables, losque han aumentado la disponibilidad a través de productos procesados a precios más bajos que las opciones saludables (12), permitiendo un mayor acceso a alimentos con alto contenido en grasas saturadas, sal, azúcares y densidad energética(13).

Los estudiantes universitarios son una población vulnerable en lo económico, social y alimentario, ya que en esta etapa asumen entre otros aspectos la responsabilidad en la alimentación, la que por factores asociados al uso del tiempo, comodidad y accesibilidad de opciones poco saludables a bajo costo, optan por la comida rápida y preparada, lo que contribuye a la formación de malos hábitos alimentarios, que a largo plazo afectan el estado nutricional $(14,15)$. Esta alimentación se caracteriza por poseer una alta densidad energética, proveniente de azúcares simples y grasas, siendo insuficiente en nutrientes esenciales para la salud como los ácidos grasos mono y poliinsaturados y la fibra dietética factores que pueden afectar la salud cardiovascular (16-19).

El objetivo de este estudio fue describir los hábitos alimentarios de estudiantes universitarias de primer año, de sexo femenino de la Universidad de Playa Ancha y evaluar el consumo de nutrientes críticos y protectores respecto a las recomendaciones de las Guías Alimentarias para la Población Chilena 2013 (1) a los que se agregó el consumo de alcohol y el factor de riesgo cardiovascular.

\section{MATERIAL Y METODOS}

Estudio descriptivo, transversal no experimental de los hábitos alimentarios en estudiantes de sexo femenino de las carreras Educación Física Damas (EFI), Educación Parvularia (EPA) y Nutrición y Dietética (NYD) de la UPLA.

El universo estaba constituído por 97 estudiantes correspondientes a todas las estudiantes mujeres que cursaban el primer año de las carreras señaladas, de las cuales, 52 parti-

TABLA 1

Relación entre nutrientes críticos y nutrientes protectores con los mensajes de las Guías Alimentarias de Chile, 2013. Factor de riesgo cardiovascular $\left(^{*}\right)$ y factor alimentario $\left(^{* *}\right)$

\begin{tabular}{|c|c|c|}
\hline & Nutriente & Recomendación Mensaje Guía Alimentaria de Chile, 2013 \\
\hline \multirow{4}{*}{$\begin{array}{l}\text { Nutriente crítico } \\
\text { y factor de } \\
\text { riesgo } \\
\text { cardiovascular }\end{array}$} & Sodio & "Come alimentos con poca sal y saca el salero de la mesa". \\
\hline & $\begin{array}{l}\text { Azúcares } \\
\text { simples }\end{array}$ & $\begin{array}{l}\text { "Si quieres tener un peso saludable, evita el azúcar, dulces, } \\
\text { bebidas y jugos azucarados". }\end{array}$ \\
\hline & $\begin{array}{l}\text { AGS- Col- } \\
\text { AG } \\
\text { Trans*** }\end{array}$ & $\begin{array}{l}\text { "Cuida tú corazón evitando las frituras y alimentos con grasas } \\
\text { como cecinas y mayonesa". }\end{array}$ \\
\hline & Alcohol & $\begin{array}{l}\text { Se le dio importancia a su frecuencia de consumo semanal, sin } \\
\text { importar el tipo de alcohol. }\end{array}$ \\
\hline \multirow{4}{*}{$\begin{array}{l}\text { Nutrientes } \\
\text { protectores y } \\
\text { factor } \\
\text { alimentario** }\end{array}$} & \multirow{2}{*}{$\begin{array}{l}\text { Fibra } \\
\text { Dietética** }\end{array}$} & $\begin{array}{l}\text { "Come } 5 \text { veces frutas y verduras frescas de distintos colores, } \\
\text { cada día". }\end{array}$ \\
\hline & & $\begin{array}{l}\text { "Consume legumbres al menos } 2 \text { veces por semana, sin } \\
\text { mezclarlas con cecinas". }\end{array}$ \\
\hline & $\begin{array}{l}\text { AGMI- } \\
\text { AGPI**** }\end{array}$ & $\begin{array}{l}\text { "Para mantener sano tú corazón, come pescado al horno o a la } \\
\text { plancha, } 2 \text { veces por semana". }\end{array}$ \\
\hline & Agua & "Para mantenerte hidratado, toma 6-8 vasos de agua al dia". \\
\hline
\end{tabular}

*Alcohol

** Fibra dietética.

*** Acidos grasos saturados, colesterol, ácidos grasos trans.

**** Acidos grasos monoinsaturados. Acidos grasos poliinsaturados. 
ciparon de forma voluntaria, firmando previamente un consentimiento informado. A la muestra obtenida se le aplicaron criterios de inclusión (ser estudiante regular del primer año de las carreras definidas de sexo femenino y tener edad entre $17 \mathrm{y}$ 27 años) y de exclusión (portadoras de enfermedades crónicas o que afecten a la alimentación, síndrome de mala absorción, enfermedad de Crohn y embarazo, entre otras). La muestra final fue de 50 estudiantes, de las cuales ,17 correspondieron a la carrera de EFI, 14 a EPA y 19 a NYD respectivamente. El promedio de edad de los estudiantes era de 19,8 $\pm 1,13$ años.

Se aplicó una encuesta de frecuencia de consumo cuanti- ficado, la que fue sometida a un proceso de validación previa por estudiantes y nutricionistas. Se elaboró con preguntas cerradas de selección múltiple respecto a los siguientes grupos de alimentos; cereales, lácteos, carnes, leguminosas secas, frutas y verduras, pescados y mariscos, alimentos altos en grasa, azúcares simples, bebidas alcohólicas, agua y sal. Además se incluyeron imágenes de los alimentos y preparaciones en porciones de consumo para una mayor certeza al momento de responder. Los nutrientes a evaluar se clasificaron en nutrientes críticos y nutrientes protectores de la salud CV. En los nutrientes críticos, grasas saturadas además, se evaluó el componente

TABLA 2

Clasificación del consumo de nutrientes críticos y factor de riesgo cardiovascular*.

Alto, moderado, bajo según frecuencia de consumo

\begin{tabular}{|c|c|c|c|c|}
\hline $\begin{array}{l}\text { Nutriente } \\
\text { crítico } y / 0 \\
\text { factor de } \\
\text { riesgo }\end{array}$ & Alimento & $\begin{array}{l}\text { Alto } \\
\text { Frecuencia } \\
\text { consumo }\end{array}$ & $\begin{array}{l}\text { Moderado } \\
\text { Frecuencia de } \\
\text { consumo }\end{array}$ & $\begin{array}{l}\text { Bajo } \\
\text { Frecuencia de } \\
\text { consumo }\end{array}$ \\
\hline \multirow{6}{*}{ Sodio } & $\begin{array}{l}\text { Embutidos, productos } \\
\text { fritos, aceitunas saladas, } \\
\text { bocadillos salados }\end{array}$ & $\begin{array}{l}\text { 3-4 días o más } \\
\text { semanal }\end{array}$ & $\begin{array}{l}1-2 \text { dias } \\
\text { semana }\end{array}$ & No consume \\
\hline & Frutos secos: con o sin sal & Con sal & - & Sin sal \\
\hline & \multirow{4}{*}{$\begin{array}{l}\text { Sal: Mide la sal al momento } \\
\text { de cocinar }\end{array}$} & No mide & - & Si mide \\
\hline & & 1 puñado & 1 pizca & $\begin{array}{l}\text { La punta del chuchillo } \\
\text { o } 1 \text { cucharadita }\end{array}$ \\
\hline & & Con salero en la mesa & - & Sin salero en la mesa \\
\hline & & $\begin{array}{l}\text { Agrega sal antes de } \\
\text { probar el alimento }\end{array}$ & - & $\begin{array}{l}\text { No agrega sal antes de } \\
\text { probar el alimento }\end{array}$ \\
\hline \multirow{2}{*}{$\begin{array}{l}\text { Azúcares } \\
\text { simples }\end{array}$} & \multirow{2}{*}{ Azúcar } & 4 cdtas por vez al dia & $\begin{array}{l}3 \text { cdtas por vez al } \\
\text { día }\end{array}$ & No consume \\
\hline & & 3 veces al día & 2 veces al día & $\begin{array}{l}1-2 \text { cdtas } \\
1 \text { vez al día }\end{array}$ \\
\hline \multirow{4}{*}{$\begin{array}{l}\text { Azúcares } \\
\text { agregados }\end{array}$} & Bebidas y jugos azucarados & Todos los días & $\begin{array}{l}\text { 1- } 2 \text { días a la } \\
\text { semana }\end{array}$ & No consume \\
\hline & Cereales de desayuno & $\begin{array}{l}\text { Azucarados } \\
\text { (chocapic, zucaritas, } \\
\text { estrellitas) }\end{array}$ & $\begin{array}{l}\text { Medianamente } \\
\text { azucarados } \\
\text { (adelgazul,fitness) }\end{array}$ & $\begin{array}{l}\text { Bajos en azúcar } \\
\text { (avena, quínoa) }\end{array}$ \\
\hline & Productos de pastelería & $\begin{array}{l}\text { 3-4 dias o más a la } \\
\text { semana }\end{array}$ & $\begin{array}{l}\text { 1-2 dias la } \\
\text { semana }\end{array}$ & $\begin{array}{l}\text { No consume o } 1 \text { a } 2 \\
\text { veces al mes. }\end{array}$ \\
\hline & Bocadillos dulces & $\begin{array}{l}\text { 3-4 días o más a la } \\
\text { semana }\end{array}$ & $\begin{array}{l}1-2 \text { dias a la } \\
\text { semana }\end{array}$ & No consume \\
\hline $\begin{array}{l}\text { AGS }, \text { COL } \\
\text { AG Trans** }\end{array}$ & $\begin{array}{l}\text { Embutidos, mayonesa, } \\
\text { frituras, comida chatarra }\end{array}$ & $\begin{array}{l}\text { 3-4 días o más a la } \\
\text { semana }\end{array}$ & $\begin{array}{l}\text { 1-2 dias a la } \\
\text { semana }\end{array}$ & No consume \\
\hline Alcohol & $\begin{array}{l}\text { Bebidas } \\
\text { alcohólicas(destiladas y no } \\
\text { destiladas }\end{array}$ & Todos los dias & $\begin{array}{l}1-2 \text { veces a la } \\
\text { semana }\end{array}$ & No consume \\
\hline
\end{tabular}

** Acidos grasos saturados. Colesterol. Acidos grasos trans. 
colesterol y se agregó en este grupo el consumo de alcohol, principalmente por ser un factor de riesgo cardiovascular y de aporte calórico a la dieta. En los nutrientes protectores se incluyó, el factor alimentario, la fibra dietética. Cada nutriente crítico y protector fue relacionado con 7 mensajes de los 11 que contienen las Guías Alimentarias 2013 para la población chilena (tabla 1). Se categorizó la clasificación del consumo de nutrientes críticos y factor de riesgo CV (alcohol) y nutrientes protectores y el factor alimentario (fibra dietética) en alto, moderado y bajo según la frecuencia de consumo diario o semanal de los productos seleccionados respecto a las recomendaciones de las Guías Alimentarias 2013. La baja frecuencia de consumo de nutrientes críticos indica que cumple con las recomendaciones de las Guías Alimentarias 2013, mientras que una alta frecuencia de un consumo sobre dicha recomendación, indica lo contrario.Por su parte, en los nutrientes protectores, una alta frecuencia de consumo indica un alto consumo y una baja frecuencia un bajo consumo y que no se logran las recomendaciones de las Guías Alimentarias 2013 para la población chilena (tablas 2 y 3).

Los nutrientes críticos que se consideraron fueron; sodio, azúcares simples y agregados, ácidos grasos saturados y su componente colesterol, grasas trans y factor de riesgo CV, alcohol. Los nutrientes protectores fueron los ácidos grasos mono y poliinsaturados, el agua y el factor alimentario, la fibra dietética, los que se relacionaron con los siguientes alimentos (tablas 2 y 3).

Sodio: embutidos, productos fritos salados (empanadas fritas, sopaipillas y papas fritas y churros), bocadillos salados (papas fritas envasadas, ramitas y galletas saladas), aceitunas y frutos secos con o sin sal; sal agregada al momento de cocinar, presencia del salero en la mesa y añadido de sal a los alimentos antes de probarlos. En relación a la medición de la ingesta de sal, que es de difícil evaluación, generalmente las orientaciones para estimar su consumo y la reducción se dirigen a aspectos cualitativos que complementan los antecedentes cuantitativos

\section{TABLA 3}

Clasificación del consumo de nutrientes protectores y factor alimentario ${ }^{*}$ ).

Alto, moderado, bajo según frecuencia de consumo.

\begin{tabular}{|c|c|c|c|c|}
\hline $\begin{array}{l}\text { Nutrientes } \\
\text { protectores y } \\
\text { factor } \\
\text { alimentario(*) }\end{array}$ & Clasificación & Alto & Moderado & Bajo \\
\hline \multirow{2}{*}{$\begin{array}{l}\text { Fibra } \\
\text { dietética }\end{array}$} & Frutas, Verduras & $\begin{array}{l}\text { Todos los } \\
\text { Días }\end{array}$ & $\begin{array}{l}\text { 3-6 días a la } \\
\text { semana }\end{array}$ & $\begin{array}{l}\text { 1 -2 días a la } \\
\text { semana } \\
\text { No consume }\end{array}$ \\
\hline & Legumbres & $\begin{array}{l}\text { 1-2 días o } \\
\text { más a la } \\
\text { semana }\end{array}$ & $\begin{array}{l}1-2 \text { veces por } \\
\text { mes }\end{array}$ & No consume \\
\hline \multirow{2}{*}{$\begin{array}{l}\text { AGMI- } \\
\text { AGPI** }\end{array}$} & Pescados y mariscos & $\begin{array}{l}\text { 1-2 días o } \\
\text { más a la } \\
\text { semana }\end{array}$ & - & No consume \\
\hline & Palta y aceitunas & $\begin{array}{l}\text { 3-4 dias o } \\
\text { más a la } \\
\text { semana }\end{array}$ & $\begin{array}{l}\text { 1-2 dias a la } \\
\text { semana }\end{array}$ & No consume \\
\hline Agua & $\begin{array}{l}\text { Agua potable o mineral sin } \\
\text { sabor. }\end{array}$ & $\begin{array}{l}\text { Todos los } \\
\text { días }\end{array}$ & $\begin{array}{l}\text { 5-6 días a la } \\
\text { semana }\end{array}$ & $\begin{array}{l}\text { 3-4 días } \\
\text { No consume }\end{array}$ \\
\hline
\end{tabular}

\footnotetext{
* Fibra dietética

** Acidos grasos monoinsaturados. Acidos grasos poliinsaturados
} 
obtenidos en productos procesados con alto contenido de sodio en su preparación $(20,21)$.

Azúcares simples: Azúcar como tal agregada a las infusiones té, café, leche; azúcares agregados; bebidas y jugos azucarados, cereales de desayuno (azucarados, medianamente azucarados y bajos en azúcar), productos de pastelería y bocadillos dulces (galletas y chocolates), AGS- COL - AG Trans: embutidos, productos fritos salados (empanadas fritas, sopaipillas y papas fritas, churros), bocadillos salados, mayonesa, mantequilla y margarina; y comida chatarra (completos, pizzas, churrascos y lomitos y chorrillanas). Bebidas alcohólicas destiladas y fermentadas.

TABLA 4

Distribución del consumo de nutrientes críticos y factor de riesgo cardiovascular en estudiantes universitarias según carrera. Valparaíso 2013. En porcentaje (\%).

\begin{tabular}{|c|c|c|c|c|}
\hline \multicolumn{2}{|c|}{ Nutriente crítico } & \multirow{2}{*}{$\begin{array}{l}\text { Bajo } \\
25,1\end{array}$} & \multirow{2}{*}{$\begin{array}{l}\text { Moderado } \\
36,5\end{array}$} & \multirow{2}{*}{$\begin{array}{l}\text { Alto } \\
38,4\end{array}$} \\
\hline Estudiantes & Sodio & & & \\
\hline \multirow[t]{4}{*}{ Física } & Azúcares simples & 58,8 & 32,4 & 8,8 \\
\hline & Azúcares agregados & 24,7 & 28,2 & 47,1 \\
\hline & $\begin{array}{l}\text { COL-AGS-AG } \\
\text { TRANS* }\end{array}$ & 15,3 & 43,5 & 41,2 \\
\hline & Alcohol** & 87,5 & 12,5 & 0,0 \\
\hline \multirow{5}{*}{$\begin{array}{l}\text { Estudiantes } \\
\text { Educación } \\
\text { Parvularia }\end{array}$} & Sodio & 57,1 & 10,7 & 32,1 \\
\hline & Azúcares simples & 53,6 & 32,1 & 14,3 \\
\hline & Azúcares agregados & 22,9 & 38,6 & 38,6 \\
\hline & $\begin{array}{l}\text { COL-AGS-AG } \\
\text { TRANS* }\end{array}$ & 25,7 & 48,6 & 25,7 \\
\hline & Alcohol** & 87,5 & 12,5 & 0,0 \\
\hline \multirow{5}{*}{$\begin{array}{l}\text { Estudiantes } \\
\text { Nutrición y } \\
\text { Dietética }\end{array}$} & Sodio & 46,7 & 27,1 & 26,2 \\
\hline & Azúcares simples & 68,4 & 26,3 & 5,3 \\
\hline & Azúcares agregados & 46,7 & 33,6 & 19,7 \\
\hline & $\begin{array}{l}\text { COL-AGS-AG } \\
\text { TRANS* }\end{array}$ & 16,8 & 54,7 & 28,4 \\
\hline & Alcohol ** & 100,0 & 0,0 & 0,0 \\
\hline
\end{tabular}


Nutrientes protectores: AGMI-AGPI: pescados y mariscos; palta y aceitunas y frutos secos. Agua: potable o agua mineral sin sabor y sin azúcar. Factor alimentario fibra dietética; frutas, verduras y leguminosas.

\section{RESULTADOS}

Se presentan las frecuencias de consumo de los productos seleccionados por las estudiantes universitarias de EFI, EPA y NYD respecto a las recomendaciones de las Guías Alimentarias 2013, alcohol y fibra dietética (tabla 4).

Nutrientes críticos. Sodio: Se observó que las estudiantes de EFI y EPA presentan un alto consumo de sodio $(38,4 \%)$ y $(32,1 \%)$ respectivamente, lo que indica una frecuencia de consumo más de tres veces de los productos seleccionados. Destaca el bajo consumo en las estudiantes de EPA. Azúcares simples y agregados. En las estudiantes de las tres carreras se observó una baja frecuencia de consumo de azúcares simples (1 vez al día con 1 a 2 cdtas), destacan los estudiantes de NYD con $68,4 \%$ de bajo consumo y a la vez presentan $19,7 \%$ de alto consumo en azúcares agregados provenientes de bebidas, jugos azucarados, cereales azucarados y productos de pastele- ría. En AGS, AG Trans y COL, se observó un 43,5\%, 48,6\% y $54,7 \%$ en las estudiantes de EFI, EPA y NYD respectivamente con una frecuencia de $1-2$ veces a la semana de embutidos, productos fritos, mayonesa, margarina o mantequilla.Alcohol. Se observó un bajo consumo de alcohol de las estudiantes de las tres carreras con una frecuencia de consumo de 1 a 2 veces a la semana.

Nutrientes protectores. Fibra dietética. Se detectó que $45,1 \%$, de las estudiantes de EFI presentaban un alto consumo de fibra dietética con una frecuencia de consumo de leguminosas igual o superior a 2 veces a la semana y diaria de frutas y verduras. A su vez, 47,6\% y $40 \%$ de las estudiantes de EPA y de NYD, respectivamente, tenían un moderado consumo de fibra dietética con una frecuencia de consumo de leguminosas menor a 1-2 días a la semana y entre de 3 a 6 días a la semana de frutas y verduras (tabla 5).

AGMI-AGPI: se observó que 42,6\%; 39,2\% y 36,8\% de las estudiantes de EFI, EPA y NYD respectivamente clasificaron en bajo consumo lo que señala que no consumen pescados, mariscos, paltas y aceitunas. Sólo 29,4 \%, 34,1\% y $36,8 \%$ en las estudiantes de EFI, EPA y NYD respectivamente

TABLA 5

Distribución del consumo de nutrientes protectores y factor alimentario $\left(^{*}\right)$ en estudiantes universitarias según carrera. Valparaíso 2013. En porcentaje (\%).

\begin{tabular}{|l|l|l|l|l|}
\hline \multicolumn{2}{|l|}{$\begin{array}{l}\text { Nutriente protector y factor } \\
\text { alimentario* }\end{array}$} & Bajo & Moderado & Alto \\
\hline $\begin{array}{l}\text { Estudiantes } \\
\text { Educación } \\
\text { Física }\end{array}$ & Fibra Dietética & 31,4 & 23,5 & 45,1 \\
\cline { 2 - 5 } & AGMI-AGPI** & 42,6 & 27,9 & 29,4 \\
\cline { 2 - 5 } & Agua & 52,9 & 5,9 & 41,2 \\
\hline $\begin{array}{l}\text { Estudiantes } \\
\text { Educación } \\
\text { Parvularia }\end{array}$ & Fibra Dietética & 28,6 & 47,6 & 23,8 \\
\cline { 2 - 5 } & AGMI-AGPI** & 39,5 & 26,4 & 34,1 \\
\cline { 2 - 5 } & Agua & 64,3 & 7,1 & 28,6 \\
\hline $\begin{array}{l}\text { Estudiantes } \\
\text { Nutrición y } \\
\text { Dietética }\end{array}$ & Fibra Dietética & 28,1 & 40,4 & 31,6 \\
\cline { 2 - 5 } & AGMI-AGPI** & 36,8 & 26,3 & 36,8 \\
\cline { 2 - 5 } & Agua & 52,6 & 0,0 & 47,4 \\
\hline
\end{tabular}

\footnotetext{
* Fibra dietética.

** Acidos grasos monoinsaturados. Acidos grasos poliinsaturados.
} 
presentaron alto consumo, con una frecuencia de 2 veces a la semana.

Agua potable o mineral sin sabor. Se observó que 52,9\%, $64,3 \%$ y $52,6 \%$ de las estudiantes de EFI, EPA y NYD simultáneamente, clasificaron en bajo consumo de agua potable o mineral, es decir, con una frecuencia de consumo 3 a 4 veces a la semana o menor. Menos de la mitad de las estudiantes toman agua todos los días.

A modo general, se observó que las estudiantes de EFI presentaron un alto consumo de nutrientes críticos sodio, azúcares agregados; a su vez, las estudiantes de EPA y END un bajo consumo. Las estudiantes de las tres carreras presentaron un moderado consumo de AGS,COL, AGTrans.

En relación a los nutrientes protectores se observó que las estudiantes de las tres carreras presentaron una frecuencia de consumo menor a las recomendaciones de las guías alimentarias.

\section{DISCUSIÓN}

En los nutrientes críticos y factor de riesgo, destaca el alto consumo de sodio que en Chile es 9,8 g al día (6), superando la recomendación establecida por la OMS de 5 g diarios $(20,21)$. Por lo tanto, las estrategias de reducción del consumo de sal en toda la población son necesarias para disminuir los riesgos asociados a las ECV (1). El consumo de sodio es bajo para dos de las carreras, EPA y NYD lo que podría indicar que siguen los lineamientos del mensaje de las guías alimentarias para la población chilena: "Come alimentos con poca sal y saca el salero de la mesa", miden la sal al momento de cocinar, utilizando la punta de un cuchillo o una cucharadita de té (1 g y $5 \mathrm{~g}$ de sal aprox. respectivamente). Cabe destacar que 1 $\mathrm{g}$ de sal equivale a $390 \mathrm{mg}$ de sodio. Además no añaden sal a los alimentos sin antes de probarlos y sacan el salero de la mesa, mensajes orientados para reducir el consumo de sodio diariamente. En relación a la medición del consumo de sal, las orientaciones tanto para estimar su consumo y reducción se dirigen a aspectos cualitativos para observar las actitudes y conductas respecto a la cantidad de sal añadida en la mesa y al cocinar $(20,21)$.

Los azúcares agregadosal ser de rápida absorción y de bajo poder de saciedad favorecen una mayor ingesta calórica condicionando el aumento de la $\mathrm{OB}$, diabetes, enfermedades CV y cáncer (1).

Los alimentos ricos en grasas y los productos fritos son principales fuentes de AGS-COL y AG Trans que consumidos por sobre la recomendación $(22,23)$ favorecen su depósito en el abdomen, arterias coronarias, siendo factores de riesgo CV al igual que los AG Trans que actúan sobre las lipoproteínas plasmáticas con un incremento del LDL y un descenso del HDL aumentando así, el riesgo de enfermedades CV (24-27). Las estudiantes de las tres carreras analizadas presentan un bajo consumo de alcohol, contrastando con estudios donde se evidencia su alto consumo en estudiantes universitarios $(15,17)$. El consumo de alcohol es un factor a considerar en los jóvenes dado el inicio de su consumo cada vez a menor edad y por el efecto que tiene sobre la salud CV y las enfermedades hepáticas (28-30).

Los resultados obtenidos en la aplicación de la encuesta de frecuencia de consumo señalan que en relación a los nutrientes críticos las estudiantes de las tres carreras analizadas presentan un bajo consumo de sodio, azúcares simples, azúcares agregados y alcohol para las estudiantes de EPA y NYD. Por su parte EFI presentan frecuencias de consumo superiores a las recomendadas para el sodio, azúcares agregados y ácidos grasos saturados, Trans y colesterol. Esta situación es similar a la encontrada en otros estudios realizados en estudiantes universitarias que señalan un consumo por sobre la recomendación de estos nutrientes $(18,24)$.

Respecto a los nutrientes protectores, se evidencia un consumo bajo la recomendación de las Guías Alimentarias para el factor alimentario fibra dietética, lo que indica una frecuencia de consumo para frutas-verduras y leguminosas entre 3-6 días y menos de 2 días por semana, respectivamente. De igual forma, para el consumo de AGMI-AGPI en las tres carreras se observó una baja frecuencia de consumo. Diversos autores, del mismo modo, han referido que el consumo de frutas, verduras y legumbres no es suficiente en estudiantes universitarios $(22,24)$, señalando que el consumo de fibra dietética no logra la recomendación diaria $(3,7,15)$. Otro nutriente que presenta un bajo consumo, es el agua, en el cual las estudiantes no alcanzan la recomendación de consumo de las Guías Alimentarias durante la semana o bien, lo hacen entre 3 a 4 veces (o menos), con resultados similares en otros estudios donde la ingesta de agua no alcanza dicha recomendación $(1,28,30)$.

Los resultados observados sugieren que de mantenerse este perfil, se podrían producir efectos perjudiciales para la salud, ya que un bajo consumo de fibra dietética favorece el desarrollo de $O B$, síndrome metabólico, DM, enfermedades CV, y algunos tipos de cánceres (31-34).En la Guía para Universidades Saludables y Guías Alimentarias para la población de Chile $(1,35)$ se establece que la promoción de la salud es prioritaria, y que es fundamental incorporar más opciones adecuadas en alimentación que contribuyan a mejorar los hábitos alimentarios de los estudiantes universitarios mejorando el acceso a un menú variado, atractivo y accesible, lo que contribuiría a mejorar las conductas alimentarias de los universitarios.

Se sugiere la incorporación en los planes de estudios en la educación superior la asignatura de Promoción de la Salud y Estilos de Vida Saludables que contemple alimentación saludable dando a conocer la influencia de los nutrientes críticos y protectores en la salud cardiovascular. En este sentido, las Guías Alimentarias para la población chilena, son una herramienta práctica, de fácil aplicación para evaluar la ingesta de nutrientes, educar y orientar las conductas alimentarias $(21,22)$, por lo que se debería realizar una mayor difusión de las Guías Alimentarias como un instrumento para la educación alimentaria y nutricional para la población universitaria.

\section{LIMITANTES DEL ESTUDIO}

Corresponde a una muestra pequeña de estudiantes de tres carreras universitarias, por lo que no se pueden extrapolar los resultados a todas las estudiantes de educación superior de esta casa de estudios.

Los criterios usados para las clasificaciones de frecuencia de consumo respecto a las guías alimentarias fueron definidos por los investigadores y pueden servir de base para otros estudios que consideren el uso de estas guías no sólo para educar sino para la vigilancia alimentaria nutricional en grupos de población.

\section{RESUMEN}

El aumento de los factores de riesgo cardiovascular en mujeres jóvenes amerita conocer su perfil nutricional. El objetivo del presente estudio fue conocer el consumo alimentario de 50 universitarias voluntarias de educación física (EFI), educación parvularia (EPA) y nutrición y dietética (NYD) de la Universidad 
de Playa Ancha, Valparaíso, Chile. Se usó un cuestionario de frecuencia para conocer consumo de nutrientes críticos (sodio, azúcares, ácidos grasos saturados, trans, colesterol y además de alcohol, factor riesgo cardiovascular) y protectores (ácidos grasos protectores mono y poliinsaturados, agua, fibra dietética). La frecuencia del consumo se clasificó en alto, moderado, bajo. Los nutrientes críticos y protectores fueron comparados con las guías alimentarias de Chile 2013. Resultados: EFI, fue alto en sodio y fibra dietética; EFI, EPA alto en azúcares agregados y bajo en agua. EFI, EPA NYD estuvieron bajos en ácidos grasos mono y poliinsaturados, moderado en ácidos grasos saturados, trans y colesterol y el alcohol bajo en los tres grupos. Lo observado, no consigue las recomendaciones de consumo de las guías alimentarias.

Palabras clave: Hábitos nutricionales, nutrientes, estudiantes universitarios, factores de riesgo cardiovascular.

Agradecimientos: Los autores agradecen a Gloria Arlegui M., Directora del Sistema de Biblioteca UPLA y a Lina Besaccia P., Bibliotecaria del Centro de Estudios Avanzados CEA, UPLA por los aportes en la revisión bibliográfica.

\section{BIBLIOGRAFÍA}

1. Ministerio de Salud. Estudio para la revisión y actualización de las guías alimentarias para la población chilena. Resolución Exenta N²60. Santiago, Chile: MINSAL; 2013 Mayo.

2. Araya $M$, De Pablo S, Olivares $S$, Reyes $M$, Rodríguez $L$, Uauy $R$, Vera $G$, Zacarías I. Propuesta de criterios y recomendación de límites máximos de nutrientes críticos para la implementación de la ley de composición de alimentos y su publicidad. Informe Técnico INTA; 2011, Santiago, Chile.

3. Crovetto $M$, Uauy $R$. Cambios en el consumo aparente de nutrientes en el Gran Santiago 1988-1997 en hogares según ingreso y su probable relación con patrón de enfermedades crónicas no transmisibles. Rev Med Chil. 2010; 138: 1091-108.

4. Bernui I, Sáez Y. Prevalencia de factores de riesgo cardiovascular en adolescentes de instituciones educativas. An. Fac. Med.2009; 70(4).Disponible en www.redalyc.org/ pdf/379/37912407006.pdf

5. Ministerio de Salud. Encuesta Nacional de Salud 20092010. Tomo I. Disponible en: http://web.minsal.cl/portal/ url/item/bcb03d7bc28b64dfe040010165012d23.pdf

6. MacMillan N. Valoración de hábitos de alimentación, actividad física y condición nutricional en estudiantesde la Pontificia Universidad Católica de Valparaíso. RevChilNutr.2007; 34(4):330-6.

7. Ministerio de Salud. Resultados I Encuesta de Salud. Santiago, Chile. MINSAL; 2003.

8. Vio F. Prevención de la obesidad en Chile. Rev Chil Nutr. 2005; 32(2): 80-7.

9. Crovetto $M$, Uauy $R$. Recomendaciones para la prevención del cáncer dadas por el Fondo Mundial para la investigación sobre cáncer (FM/C): análisis de la situación en Chile. Rev Med Chil. 2013; 141: 626-36.

10. Organización Mundial de la Salud. Estrategia Mundial sobre régimen alimentario, actividad física y salud. Resolución WHA 55.23. Disponible en: wWw.who.int/dietphysicalactivity/strategy/.../strategy_spanish_web.pdf

11. Instituto Nacional de Estadísticas. Resultados VI encuesta de presupuestos familiares Nov. 2006 - Oct. 2007. Disponible en: www.ine.cl/.../encuestas_presupuestos_familiares/VI_ENCUESTA/.../EPF

12. Barría $R$, Amigo H. Transición nutricional: una revisión del perfil latinoamericano [Internet]. Santiago, Chile: Universidad de Chile; 2006 [citado: 2014 Jun 6]. Disponible en: http://www.captura.uchile.cl/handle/2250/5406

13. Rodriguez J, Agulló T E. Estilos de vida, cultura, ocio y tiempo libre de los estudiantes universitarios. Psicotema 1999;11(2): 247-59. Disponible en www.psicothema. com/pdf/204.pdf

14. Durán S, Castillo M, Vio F. Diferencias en la calidad de vida de estudiantes universitarios de diferente año de ingreso del campus Antumapu. Rev Chil Nutr. 2009; 36(3):200-9.

15. Aller $R$, Conde $R$, De Luis D, González Sagrado $M$, LedoVarela $M$. Características nutricionales y estilo de vida en universitarios. Nutr Hosp. 2011;26(4):814-8.

16. Bazaez G, Berlanga M, Durán A, Encina C, Figueroa $K$. Comparación en la calidad de vida y estado nutricional entre alumnos de nutrición y dietética y de otras carreras universitarias de la Universidad Santo Tomás de Chile. Nutr Hosp. 2012; 27(3):739-46.

17. Espinoza O, Gálvez J, MacMillan N, Rodríguez F. Hábitos de alimentación y actividad física en estudiantes universitarios. Rev Chil Nutr. 2011; 38(4):458-65.

18. Organización Mundial de la Salud. Prevención y control de las enfermedades no trasmisibles: Aplicación de la estrategia mundial. Informe de Secretaria. En: 61ª Asamblea Mundial de la Salud;2008 [Citado: 2014 Marz 28]. Disponible en www.who.int/gb/ebwha/pdf_files/WHA61-REC1/ A61_REC1-sp.pdf

19. Organización Mundial de la Salud. Organización Panamericana de salud. Foro de la OMS sobre la Reducción del Consumo de Sal en la Población (2006, París).5-7 de Octubre del 2006, París (Francia)

20. Institute of Medicine. (2010) Strategies to Reduce Sodium Intake in the United States. Washington, DC: TheNational Academies Press. Disponible en www.ncbi.nlm.nih.gov

21. Fuentes $L$, Muñoz A. Efectos de una intervención integral en la reducción de factores de riesgo cardiovascular en mujeres con sobrepeso u obesidad de la Región de Los Ríos. Rev Med Chil. 2010; 138: 974-81.

22. Organización Mundial de la Salud (OMS).Reducción del consumo de sal en la población. Informe de un foro y reunión técnica de la OMS, del 5 al 7 de octubre del 2007, Paris, Francia.(Consultado 2014, abr 22) Disponible en: www.who.int/dietphysicalactivity/salt-report-SP.pdf

23. Álvarez $C$, Celis $C$, Flores $M$, Ramírez $R$, Zúñiga $C$. Efectos del ejercicio físico de alta intensidad y sobrecarga en parámetros de salud metabólica en mujeres sedentarias, pre-diabéticas con sobrepeso u obesidad. Rev Med Chil. 2012; 140: 1289-96.

24. Pacheco J. Hipertensión arterial en diferentes edades en la mujer. An Fac Med. 2010; 71(4):257-64.

25. Cervera F, García M, Milla M, Serrano $R$, Vico $C$. Hábitos alimentarios y evaluación alcohol nutricional en una población universitaria. Nutr Hosp. 2013; 28 (2):438-46.

26. Food and Agriculture Organization. Grasas y ácidos grasos en nutrición humana. Ginebra:FAO; 2008 p. 10 -14.

27. Organización Panamericana de la Salud. Alcohol y atención primaria de la salud: Informaciones clínicas básicas para la identificación y el manejo de riesgos y problemas. Washington DC:OPS; 2008.

28. Estruch R. Efectos del alcohol en la fisiología humana. Adicciones. 2002;14 (Supl. 1):43-61.

29. Organización Mundial de la Salud. Estrategias para reducir el uso nocivo del alcohol: proyecto de estrategia mundial. Informe de la secretaría. 63ava Asamblea Mundial de la 
Salud. Punto 11.10 del orden del día provisional A63/13.25 marzo 2010 [Internet]; [Citado: 2014 abril 22]. Disponible en: www.who.int/substance_abuse/activities/msbalcstrategyes.pdf

30. Barrón J, Domínguez M, Refugio M, Romero A. Efecto adverso en la calidad proteica de los alimentos de dietas con altos contenido de fibra dietaria. Rev Chil Nutr. 2011; 38(3): 369-75.

31. Bauce G, Cueva E, Flores Z, García P, Moya M, Peña $R$, Pérez $N$, Zulay $M$. Patrones y hábitos alimentarios: reflejo de lo que comen los jóvenes ucevistas. RFM. 2009; 32(1):69-74.

32. Hitschfeld $M$, Labbé $M$, Romero $M$, Santander J, Zamora V. Consumo de tabaco y alcohol entre los estudiantes de medicina de la Pontificia Universidad Católica de Chile. Rev Méd Chil. 2009; 137: 361-8.

33. Bermejo L, López A, Marín L, Ortega R, Perea J, Rodríguez E. Hábitos alimentarios y su relación con los conocimientos, respecto al concepto de dieta equilibrada, de un colectivo de mujeres jóvenes con sobrepeso/obesidad. Nutr. Hosp. 2007; 22(6): 654-60.

34. Crovetto, M, Uauy R.Recomendaciones para la prevención del cáncer dadas por el Fondo Mundial de Investigación sobre el Cáncer (FMIC) Análisis de la situación en Chile: Rev Méd Chil 2013;141: 626-36.

35. Lange I, Vio F. Guía para universidades saludables y otras Instituciones de educación superior. Santiago, Chile: UC y PUC.; 2006 p. 50. 